\title{
CLINICOPATHOLOGICAL FEATURES OF NONSPECIFIC INVASIVE BREAST CANCER ACCORDING TO ITS MOLECULAR SUBTYPES
}

\author{
M. Zavyalova, ${ }^{1,2,3}$, S. Vtorushin ${ }^{1,2}$, N. Telegina ${ }^{2}$, N. Krakhmal, ${ }^{1,2}$ O. Savelieva ${ }^{1,3, *}$, L. Tashireva ${ }^{1}$, \\ O. Bragina ${ }^{4}$, E. Denisov ${ }^{3,4}$, E. Kaigorodova ${ }^{1,3}$, E. Slonimskaya ${ }^{5,6}$, E. Choynzonov ${ }^{6,7}$, V. Perelmuter ${ }^{1,2}$ \\ ${ }^{1}$ Department of Pathological Anatomy and Cytology, Tomsk Cancer Research Institute, Tomsk 634050, Russian Federation \\ ${ }^{2}$ Department of Pathological Anatomy, Siberian State Medical University, Tomsk, Russian Federation \\ ${ }^{3}$ Laboratory of Translational Cell and Molecular Biomedicine, National Research Tomsk State University, \\ Tomsk, Russian Federation \\ ${ }^{4}$ Laboratory of MolecularOncologyand Immunology, Tomsk Cancer Research Institute, Tomsk634050, Russian Federation \\ ${ }^{5}$ Department of General Oncology, Tomsk Cancer Research Institute, Tomsk 634050, Russian Federation \\ ${ }^{6}$ Department of Oncology, Siberian State Medical University, Tomsk, Russian Federation \\ ${ }^{7}$ Department of Head and Neck Cancer, Tomsk Cancer Research Institute, Tomsk 634050, Russian Federation
}

The aim of the present study was to investigate the clinical and morphological features of nonspecific invasive breast cancer according to its molecular subtypes. Materials and Methods: 163 women with nonspecific invasive breast cancer (T1-4N0-3M0) were included in the present study. Luminal A type of breast cancer was detected in 101 women, luminal B type - in 23 women, overexpression of HER2/neu was identified in 14 women and triple-negative cancer - in 25 women. Results: The study revealed that various molecular subtypes of breast cancer differ in the morphological structure, the expression characteristics of the primary tumor and the rate of lymphogenous and hematogenous metastasis. Lymphogenous metastases were more frequently (in 71\%) detected in HER2/neu overexpressing breast cancer than in luminal A (41\%), luminal B (39\%) and triple-negative tumors (40\%). Hematogenous metastasis did not depend on the morphological structure of carcinoma infiltrative component, the state of tumor stroma as well as the proliferative activity in all the investigated groups. Conclusion: The revealed clinicopathological characteristics of different molecular subtypes of invasive breast cancer allow to predict the possible outcome of the disease and select personalized treatment strategy for patients more reasonably.

Key Words: invasive breast cancer, molecular subtype, HER2/neu, triple-negative cancer, intratumor heterogeneity.

In recent years the molecular genetic classification of breast tumors offered by C.M. Perou et al. (2000) is used to determine appropriate treatment for patients on an individual basis [1]. This classification is based on cluster analysis of 465 genes and enables to differentiate between the following breast cancer subtypes: luminal A, luminal B, HER2/neu-positive overexpression and triple-negative. Each molecular genetic subtype is associated with specific molecular and clinical signs and is characterized by features of tumor response to chemotherapy, metastatic behavior and different outcomes [2, 3].

Luminal A tumors are highly sensitive to hormone therapy, have low risk of recurrence and high survival rates $[2,4,5]$. Luminal $B$ tumors in comparison with luminal $A$ ones are often accompanied by metastases in lymph nodes and recurrence risk. These tumors are usually not sensitive to chemotherapy and hormone therapy, but they are sensitive to trastuzumab in cases with positive HER2/neu expression [6, 7].

HER2/neu-positive breast cancer is characterized by the large size of the primary tumor, frequent involvement of lymph nodes in the metastatic process and low survival rates. HER2/neu-positive tumors are not

Submitted: April 21, 2016.

*Correspondence: $\quad$ Fax: +73822 41-80-60;

E-mail: olga.sav.1980@gmail.com

Abbreviations used: CK - cytokeratin, EGFR1 - epidermal growth factor receptor; ER - estrogen receptors; PR - progesterone receptors. sensitive to hormone therapy, however administration of trastuzumab in the adjuvant setting is effective for them [6-8].

Triple-negative cancer is found among younger women and is often associated with BRCA1 mutations. Low differentiation grade, necrosis and inflammatory infiltration in the tumor stroma characterize the histological structure of this cancer subtype. These tumors have big size, they often metastasize to lymph nodes and distant organs and have lower survival rates. Triple-negative tumors are sensitive to anthracyclinetaxane-based chemotherapy $[9,10]$.

Classification of breast cancers described above is still relevant. Evaluation of estrogen receptors (ER), progesterone receptors (PR), HER2/neu and Ki-67 expression is considered to be the gold standard in immunohistochemical diagnosis of breast cancer. In cases of triple-negative cancer cytokeratins CK5/6 and epidermal growth factor receptor 1 or vimentin are further detected. Knowledge of this biomarker status enables to determine therapeutic schemes for breast cancer patients $[6,7,11,12]$. However, using this biomarker panel in some cases leads to considerable difficulties in interpretation of immunohistochemical reactions. It may be explained by tumor heterogeneity, which underlies simultaneous detection of luminal and basal signs. It is also the reason for distinguishing the so-called "basal-and-luminal" tumors which are considered to have cell clones responding to treat- 
ment differently. This expression peculiarity deserves special attention [13].

In addition, detailed analysis of tumor morphological structure in different molecular subtypes of breast cancer is required. Recent data regarding this issue are scarce and are limited to identification of tumor histological variant and differentiation grade only.

The aim of the present study was to investigate the clinical and morphological features of nonspecific invasive breast cancer according to its molecular subtypes.

\section{MATERIALS AND METHODS}

Patients. 163 women (mean age $-55.9 \pm$ 11.1 years) with nonspecific invasive breast cancer $\mathrm{T} 1$ 4NO-3M0, who were treated in General Oncology Department of Tomsk Cancer Research Institute (Tomsk, Russia) from January 1999 to January 2007, were included in the present study (Table 1).

Table 1. Clinicopathological characteristics of breast cancer patients

\begin{tabular}{|c|c|c|c|c|}
\hline \multirow[b]{2}{*}{ Characteristics } & \multicolumn{4}{|c|}{ Tumorsubtype } \\
\hline & $\begin{array}{l}\text { Luminal } A, \% \\
\qquad(n=101)\end{array}$ & $\begin{array}{l}\text { Luminal } B, \% \\
\qquad(n=23)\end{array}$ & $\begin{array}{c}\text { Triple-nega- } \\
\text { tive, } \% \\
(n=14)\end{array}$ & $\begin{array}{l}\text { HER2/neu- } \\
\text { positive, } \% \\
(\mathrm{n}=25) \\
\end{array}$ \\
\hline Mean age, years & $55.9 \pm 10.5$ & $53.4 \pm 12.1$ & $59.1 \pm 11.7$ & $53.7 \pm 12.3$ \\
\hline $\begin{array}{l}\text { Menopausal sta- } \\
\text { tus } \\
\text { Tumor localization }\end{array}$ & 66 & 65 & 76 & 64 \\
\hline Outer quadrants & 54 & 71 & 63 & 60 \\
\hline $\begin{array}{l}\text { Internal quad- } \\
\text { rants } \\
\text { ER }\end{array}$ & 46 & 29 & 37 & 40 \\
\hline Positive & 9 & 87 & 0 & 0 \\
\hline $\begin{array}{l}\text { Negative } \\
\text { PR }\end{array}$ & 91 & 13 & 100 & 100 \\
\hline Positive & 11 & 74 & 0 & 0 \\
\hline $\begin{array}{l}\text { Negative } \\
\text { HER2 }\end{array}$ & 89 & 26 & 100 & 100 \\
\hline Positive & 0 & 0 & 0 & 100 \\
\hline $\begin{array}{l}\text { Negative } \\
\text { Ki-67 }\end{array}$ & 100 & 100 & 100 & 0 \\
\hline Expression $\geqslant 20 \%$ & 0 & 100 & 52 & 50 \\
\hline $\begin{array}{l}\text { Expression }<20 \% \\
\text { Tumor stage }\end{array}$ & 100 & 0 & 48 & 50 \\
\hline 0 & 0 & 0 & 0 & 0 \\
\hline l & 33 & 22 & 4 & 0 \\
\hline II & 54 & 65 & 80 & 64 \\
\hline III & 9 & 13 & 16 & 36 \\
\hline IV & 4 & 0 & 0 & 0 \\
\hline \multicolumn{5}{|l|}{ Tumor size $(\mathrm{cm})$} \\
\hline $\mathrm{T} 1(<2)$ & 52 & 43 & 12 & 14 \\
\hline T2 (2-5) & 40 & 52 & 84 & 64 \\
\hline $\mathrm{T} 3(>5)$ & 8 & 5 & 4 & 22 \\
\hline \multicolumn{5}{|l|}{ Node status } \\
\hline NO & 59 & 61 & 60 & 29 \\
\hline N1 & 24 & 26 & 8 & 43 \\
\hline N2 & 11 & 7 & 20 & 7 \\
\hline N3 & 6 & 6 & 12 & 21 \\
\hline \multicolumn{5}{|c|}{ Lymph node involvement } \\
\hline Positive & 41 & 39 & 40 & 71 \\
\hline $\begin{array}{l}\text { Negative } \\
\text { Histological grade }\end{array}$ & 59 & 61 & 60 & 29 \\
\hline $\begin{array}{l}\text { Low grade } \\
\text { (I and II) }\end{array}$ & 98 & 91 & 96 & 86 \\
\hline High grade (III) & 2 & 9 & 4 & 14 \\
\hline
\end{tabular}

The study was approved by the Local Medical Ethics Committee, and informed consents were obtained from all the patients prior to analysis. The patients did not receive preoperative treatment. 99 (61\%) patients went through radical mastectomy, and 64 (39\%) women underwent sectoral resection of breast cancer with axillary lymph node dissection. In the postoperative period 122 women received adjuvant chemotherapy schemes
CMF (cyclophosphamide + methotrexate + fluorouracil), FAC (fluorouracil + doxorubicin + cyclophosphamide), CAX (cyclophosphamide + doxorubicin + capecitabine). Antiestrogen therapy with tamoxifen was prescribed for 41 postmenopausal women. Postoperatively 40 patients were treated with remote gamma-therapy on the postoperative scar and/or regional lymph outflow zones in the standard mode. The period of patient monitoring was from 5 to 10 years.

Methods. Morphological examination of the surgical specimens was performed by the standard method using a light microscope "Carl Zeiss Axio Lab.A1". The histological type of breast cancer was defined according to recommendations of the World Health Organization (Geneva, 2012). The study included only cases with nonspecific invasive carcinoma. The malignancy grading was estimated according to the modified Scarff - Bloom - Richardson grading system. Previously we described the diversity of invasive growth patterns of tumor cells probably resulted in high intratumor morphological heterogeneity, which (e.g. in breast cancer) is represented by different morphological structures: tubular, alveolar, trabecular, solid structures (patterns), and discrete (small) groups of tumor cells [14-17].

Expression of hormone receptors and oncoproteins was assessed by immunohistochemistry: DAKO Clone 1D5 was used for ER, DAKO Clone PgR 636 - for PR and DAKO Clone MIB-1 - for Ki-67. Expression of sex hormone receptors was determined by HistoScore. Assessment of Ki- 67 was based on the percentage of positive cell nuclei in each variant of parenchymal component structures of primary nonspecific invasive carcinoma, regardless of staining intensity.

HER2/neu protein overexpression was determined by immunohistochemistry: DAKO polyclonal rabbit anti-human antibodies were used for c-erB-2 (dilution $1: 500$ ) and it was semi-quantitated based on staining of the cytoplasmic membrane rather than cytoplasm itself. HER2/neu overexpression was rated negative $(0+$ and $1+$ ), indeterminate $\left(2^{+}\right)$or positive $(3+)$. In cases of $2+$, women were not included in the study. The positivity cut-off value for HER2/neu was set at $30 \%$.

For the purpose of the present study breast cancer was classified into four subtypes based on estrogens and progesterone hormone receptors, HER2 and Ki-67 values: luminal A (ER ${ }^{+} \mathrm{PR}^{+} \mathrm{HER} 2^{-}$, Ki-67 expression $<20 \%$ ), luminal B (ER+PR+HER2 ${ }^{-}$, Ki-67 expression $\geqslant 20 \%$ ), HER2/neu-positive overexpression (ER-PR-HER2 ${ }^{+}$) and triple-negative (ER-PR-HER2-). Luminal A type of breast cancer was detected in 101 women, luminal B type - in 23 women, overexpression of HER2/neu - in 14 women and triplenegative cancer - in 25 women.

The presence of metastatic lesions was assessed in the lymph nodes, and the number of lymph nodes with metastases was counted. Information about the frequency and timing of hematogenous metastasis was received from patient case histories or outpatient cards. 
Statistical analysis. Qualitative variables were expressed as " $n$ " and percentage, whereas quantitative variables were expressed as their mean $(\mathrm{M})$ value and standard deviation (SD). In order to compare qualitative variables, the $x^{2}$-test was implemented. Mean values were compared using ANOVA. Statistical significance was set at $p<0.05$, using two-tail approach. Statistical analysis was performed using STATISTICA 8.0 for Windows (StatSoft Inc., USA).

\section{RESULTS}

Such clinical parameters as age and menstrual status of women were analyzed in the present study. The mean age of patients with various breast cancer molecular subtypes did not differ (luminal A subtype $-55.9 \pm 10.5$ years, luminal B subtype $-53.4 \pm$ 12.1 years, triple-negative cancer $-59.1 \pm 11.7$ years and HER2/neu overexpressing tumors $-53.7 \pm$ 12.3 years). In all the investigated groups of patients menopausal status was as follows: in patients with luminal A subtype - in $66 \%$ of cases; with luminal B subtype - in $65 \%$ of cases; with triple-negative cancer - in $76 \%$ of cases; with HER2/neu overexpression - in $64 \%$ of cases. Unilateral lesion of the breast was detected in most cases. Tumors were more frequently localized in the outer quadrants (luminal A tumors $-54 \%$, luminal B tumors $-71 \%$, triplenegative cancer - 63\%, HER2/neu overexpressing tumors $-60 \%)$. The detection rate of multicenter tumor growth did not differ in all the patient groups as well (luminal $A-21 \%$, luminal B $-22 \%$, triple-negative cancer $-13 \%$ HER2/neu overexpression $-21 \%$ ).

Small tumors $(<2 \mathrm{~cm}$ in diameter) were more prevalent in patients with luminal cancer types, whereas larger tumors were typical of patients with triple-negative and HER2/neu overexpressing tumors (Fig. 1).

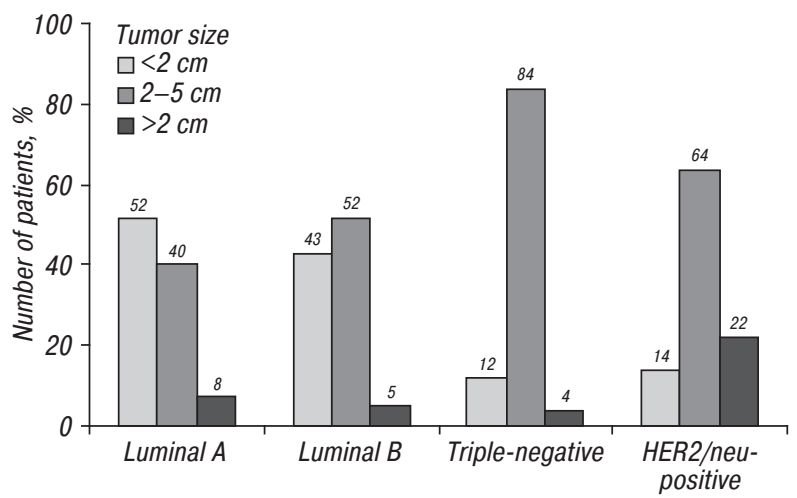

Fig. 1. Tumor size in patients with various molecular breast cancer subtypes

The morphological examination of the primary tumor in most cases revealed the second grade of malignancy: in $90 \%$ of cases in luminal A breast cancer, in $80 \%$ of cases in luminal B subtype, in $95 \%$ of cases in triple-negative and in $86 \%$ of cases in HER2/neu overexpressing tumors. The frequency of ductal structure detection did not differ in the investigated groups.

The histological investigation of primary tumor infiltrative component revealed a number of features specific to each molecular breast cancer subtype. It turned out that the infiltrative component in luminal $A$ and $B$ molecular subtypes was very diverse, all five types of the structures classified by us (alveolar, trabecular, tubular, solid and discretely spaced groups of tumor cells) were detected in them more often (in 13 and 22\% of cases, respectively). In the meantime, histological monomorphic neoplasms were detected in triplenegative and HER2/neu overexpressing tumors (in 16 and $14 \%$ of cases, accordingly), the infiltrative component in them had only one variant of structures. The received data demonstrate different grades of intratumoral morphological heterogeneity in women with different breast cancer molecular subtypes (Table 2).

Table 2. Number of different structure types in the infiltrative component of nonspecific invasive carcinoma according to the molecular subtypes of breast cancer

\begin{tabular}{ccccc}
\hline $\begin{array}{c}\text { Number of struc- } \\
\text { ture types in the } \\
\text { infiltrative com- } \\
\text { ponent }\end{array}$ & $\begin{array}{c}\text { Luminal A } \\
(\mathrm{n}=101)\end{array}$ & $\begin{array}{c}\text { Luminal B } \\
(\mathrm{n}=23)\end{array}$ & $\begin{array}{c}\text { Triple-nega- } \\
\text { tive }(\mathrm{n}=25)\end{array}$ & $\begin{array}{c}\text { HER2/neu- } \\
\text { positive } \\
(\mathrm{n}=14)\end{array}$ \\
\hline 1 & $4 / 101$ & $1 / 23$ & $4 / 25$ & $2 / 14$ \\
& $(4)$ & $(4)$ & $(16)$ & $(14)$ \\
& & & $p_{1}=0.01$ & $p 1=0.05$ \\
& & & $p_{2}=0.08$ & \\
2 & $17 / 101$ & $4 / 23$ & $6 / 25$ & $4 / 14$ \\
3 & $(17)$ & $(17)$ & $(24)$ & $(29)$ \\
& $45 / 101$ & $8 / 23$ & $8 / 25$ & $5 / 14$ \\
4 & $(44)$ & $(35)$ & $(32)$ & $(36)$ \\
& $22 / 101$ & $5 / 23$ & $6 / 25$ & $3 / 14$ \\
5 & $(22)$ & $(22)$ & $(24)$ & $(21)$ \\
& $13 / 101$ & $5 / 23$ & $1 / 25$ & $0 / 14$ \\
& $(13)$ & $(22)$ & $(4)$ & $(0)$ \\
& & & $p_{2}=0.03$ & $p_{1}=0.07$ \\
& & & & $p_{2}=0.03$ \\
\hline
\end{tabular}

Note: $p_{1}$ - compared with luminal A group; $p_{2}$ - compared with luminal $\mathrm{B}$ group.

In patients with triple-negative and HER2/neu overexpressing cancer the tubular structures were observed rarely: in 16 and $21 \%$ of cases as opposed to luminal A (45\%; $p=0.004$ and $p=0.004$, accordingly) and in luminal B (52\%; $p=0.005$ and $p=0.03$, accordingly) tumor subtypes. Our results indirectly indicate low differentiation grade of triple-negative and HER2/neu overexpressing tumors and do not conflict with data of other researchers about low-grade triple-negative cancer [18]. The other tumor structures (alveolar, trabecular, solid and discretely spaced groups of cells) were detected with approximately identical frequency.

The morphological study of the stromal tumor component showed that stromal fibrosis with parenchymal component $\leqslant 10 \%$ did not take place in triple-negative tumors in contrast to luminal $\mathrm{A}(13 \% ; p=0.02)$, luminal $\mathrm{B}(9 \% ; p=0.06)$ and HER2/neu overexpressing ( $14 \%$; $p=0.03$ ) cancer subtypes. More significant inflammatory stroma infiltration was found in triple-negative breast cancer (36\%) as opposed to luminal A ( $8 \% ; p=$ $0.0002)$ and luminal $B(13 \% ; p=0.03)$ subtypes.

The study of tumor cell proliferative activity in different breast cancer molecular subtypes showed that Ki- 67 expression in luminal A tumors was $2.00(1.00-5.00) \%$, in luminal B tumors - 20.00 (2.30-21.00)\%, in triplenegative cancer subtype $-2.00(1.00-3.00) \%$ and in HER2/neu-positive tumors - 2.50 (1.50-5.15)\%.

Lymphogenous metastases were more frequently (in $71 \%$ of cases) detected in women with HER2/neu overexpressing cancer subtype in comparison with 
luminal $\mathrm{A}(41 \% ; p=0.01)$, luminal $\mathrm{B}(39 \% ; p=0.03)$ and triple-negative tumors $(40 \% ; p=0.03)$. The number of metastatic lymph nodes in women with luminal $A$, luminal $B$ and HER2/neu overexpressing tumors did not differ $(p>0.05)$. Metastatic lesion of four and more lymph nodes (criteria N2-3) was more often diagnosed in patients with triple-negative cancer subtype (Fig. 2). The average number of regional lymph nodes involved in the metastatic process did not differ among various molecular subtypes of breast cancer $(p>0.05)$.

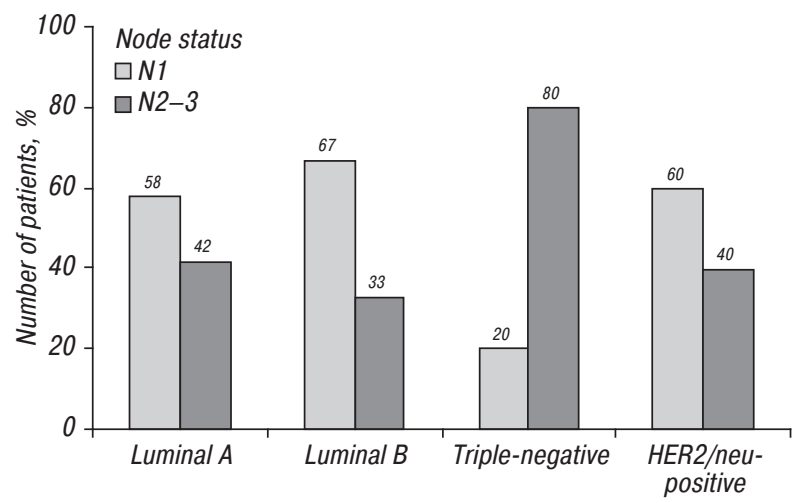

Fig. 2. Nodal metastasis rate in women with various molecular breast cancer subtypes. $p_{1}=0.01-$ compared with luminal A group; $p_{2}=0.03-$ compared with luminal $\mathrm{B}$ group; $p_{3}=$ 0.04 - compared with HER2/neu-positive group

There was no relation between the lymphogenous metastatic process and patient's age. The rate of lymphogenous metastasis was studied in various molecular breast cancer subtypes in the groups of women with different menstrual status. It was revealed that lymphogenous metastases did not occur $(0 \%)$ in the investigated patients with luminal B subtype and intact menstrual function, while in postmenopausal women it was detected in $60 \%$ of cases $(p=0.005)$.

Research of lymphogenous metastatic features depending on the morphological structure of primary tumor tissue was carried out. Among patients with luminal A and luminal B subtypes, lymphogenous metastases were more prevalent in cases with more varied morphological tumor constitution, which infiltrative component consisted of three or more different structure types. Such regularity was not observed in triple-negative and HER2/neu overexpressing tumors (Table 3).

Table 3. Lymphogenous metastasis rate in patients with various molecular breast cancer subtypes according to the number of different structure types in the infiltrative tumor component

\begin{tabular}{ccccc}
\hline $\begin{array}{c}\text { Number of struc- } \\
\text { ture types in the } \\
\text { infiltrative com- } \\
\text { ponent }\end{array}$ & $\begin{array}{c}\text { Luminal A } \\
(\mathrm{n}=101)\end{array}$ & $\begin{array}{c}\text { Luminal B } \\
(\mathrm{n}=23)\end{array}$ & $\begin{array}{c}\text { Triple-nega- } \\
\text { tive }(\mathrm{n}=25)\end{array}$ & $\begin{array}{c}\text { HER2/neu- } \\
\text { positive } \\
(\mathrm{n}=14)\end{array}$ \\
\hline 1 & $0 / 41(0)$ & $0 / 9(0)$ & $1 / 10(10)$ & $1 / 10(10)$ \\
2 & $3 / 41(7)$ & $0 / 9(0)$ & $3 / 10(30)$ & $3 / 10(30)$ \\
3 & $21 / 41(51)$ & $3 / 9(33)$ & $4 / 10(40)$ & $3 / 10(30)$ \\
& $p_{1}<0.001$ & $p_{1}=0.03$ & & \\
$4-5$ & $p_{2}<0.001$ & $p_{2}=0.03$ & & \\
& $17 / 41(42)$ & $6 / 9(67)$ & $2 / 10(20)$ & $3 / 10(30)$ \\
& $p_{1}<0.001$ & $p_{1}=0.004$ & & \\
\hline$p_{2}<0.001$ & $p_{2}=0.004$ & & \\
\hline
\end{tabular}

Note: $p_{1}$ - compared with patients with one structure type in the infiltrative component; $p_{2}$ - compared with patients with two structure types in the infiltrative component.
No significant distinctions in the incidence of hematogenous metastasis depending on patients' age, menstrual status, size and grade of tumors were revealed $(p>0.05)$ in any molecular breast cancer subtype.

Hematogenous metastasis did not depend on the morphological structure of the carcinoma infiltrative component, the state of tumor stroma as well as the proliferative activity in all the investigated groups. In women with luminal cancer subtypes the probability of hematogenous metastasis was not associated with any percentage of $\mathrm{ER}^{+}$- and $\mathrm{PR}^{+}$-cells or ER and PR expression index (on the Histo-Score scale).

The dependence of distant metastasis on status of regional lymph nodes was found only in patients with triple-negative breast cancer: all women with tumor lymph node lesion had hematogenous dissemination process $\left(\chi^{2}=9.3 ; p=0.002\right)$ at various stages of monitoring. Such regularity was not observed in other breast cancer subtypes (Fig. 3).

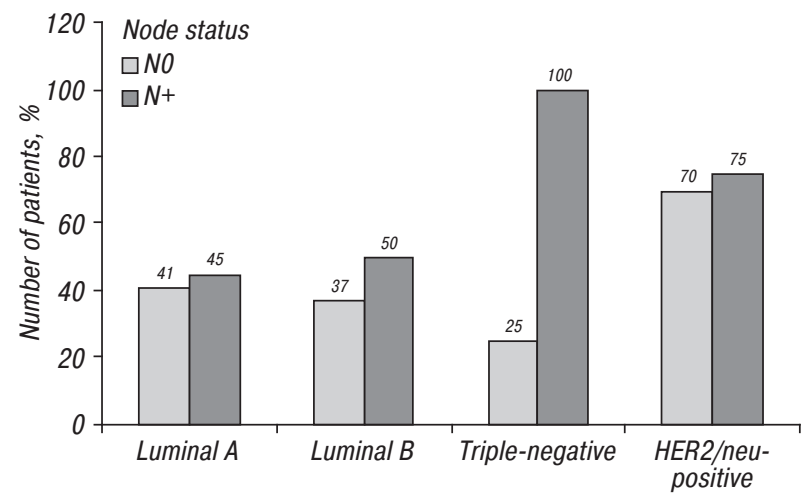

Fig. 3. Hematogenous dissemination rate according to the presence of lymph node metastases in women with various molecular breast cancer subtypes; $p=0.002-$ compared with triple-negative patients with NO node status

\section{DISCUSSION}

The present study revealed that various molecular subtypes of breast cancer differ in the morphological structure, the expression profile of the primary tumor and the rate of lymphogenous and hematogenous metastasis. It should be emphasized that these differences are found within one nosological form of nonspecific invasive breast cancer (ICD-O code 8500/3).

In our opinion one important distinction between the molecular subtypes of breast cancer is the prominence of morphological heterogeneity. According to our research, phenotypic drift takes place in nonspecific invasive cancer. It consists in the increase in time of various types of breast cancer infiltrative component structures. It turned out that luminal subtypes were often characterized by the most prominent heterogeneity. At the same time in triple-negative and HER2/neu overexpressing tumors the cases with one type of infiltrative component structures prevailed [15].

It appears that the tumor is growing faster in triplenegative and HER2/neu positive breast cancers than in the luminal subtypes: a series of indirect signs indicate it. Cases with one variant of infiltrative component structures are more common in triple-negative and HER2/neu positive breast cancers than in the luminal 
subtypes. The combination of minimal morphological structure heterogeneity with greater tumor size and greater parenchymal component volume in triplenegative cancer can be taken as an argument in favor of accelerated tumor growth in this subtype in comparison with the luminal ones.

Significant distinctions between various molecular breast cancer subtypes related to lymphogenous metastasis. In luminal subtypes of breast cancer, lymphogenous metastases were more frequent at higher morphological tumor heterogeneity. Apparently, higher morphological tumor heterogeneity, reflecting phenotypic diversity of tumor elements, makes emergence of a cell clone with lymphatic metastasis capacity more probable.

It was found that in patients with luminal B subtype the rate of lymphogenous metastases depends on the menstrual status being significantly higher in menopausal women. We have previously shown that invasive ductal breast carcinoma in women older than 35 years with intact menstrual function has significant clinicopathological and molecular genetic features as opposed to menopausal patients [19].

Triple-negative invasive breast cancer particularly differs in the parameters of lymphogenous metastasis. The lesions of four or more lymph nodes are mostly diagnosed exactly in these patients. The interest in studying lymphogenous metastatic mechanisms does not fade away. The search for the informative parameters of this process associated with the overall survival rate continues. It was agreed that an unfavorable prognostic sign is high percentage of lymph node lesions [20, 21]. However, until now the causes and the mechanisms of involvement of different number of lymph nodes in the metastatic process are unknown.

The prediction of the likelihood of axillary lymph node metastatic lesions in breast cancer in cases where sentinel lymph node is affected with metastases is a difficult problem. This problem has not been solved despite creation of several nomogram variants aiming to predict the lesion of non-sentry lymph nodes upon metastasis detection in the sentinel node $[22,23]$. In this regard, the regular involvement of a large number of lymph nodes in the metastatic process in some patients with triplenegative breast cancer may be a promising model system for studying widespread lymphogenous metastasis.

Another feature of triple-negative breast cancer is obvious relation between lymphogenous and hematogenous metastasis. Hematogenous dissemination was detected in all cases with lymph node metastatic lesion only in this molecular subtype. For other molecular subtypes of breast cancer the possibility of hematogenous metastasis did not depend on the presence of lymphogenous metastasis.

The explanation of these distinctions may be found in S. Paget' "Seed and Soil" concept [24]. It can be suggested that the emergence of tumor cell clones with the ability to metastasize ("seed") in triplenegative breast cancer subtype coincides with the formation of optimal cellular and molecular microen- vironment in the regions of hematogenous metastasis development ("soil"). Moreover, tumor cells are capable of adapting not only to the premetastatic niches in the regional lymph nodes, but, probably, to the areas of hematogenous metastases formation as well.

Apparently, triple-negative nonspecific invasive breast cancer may be a suitable model for studying the link between lymphogenous and hematogenous metastasis. The environment for emergence of "seed" and "soil" has more independent nature in luminal and HER2/neu positive breast tumors than in triplenegative cancer subtype.

Thus, different molecular genetic subtypes of breast cancer are characterized by significant morphological diversity and severity of intratumor morphological heterogeneity, which may be associated with regularity of lymphogenous and hematogenous metastasis.

\section{ACKNOWLEDGMENTS}

This work was supported by the Russian Foundation for Basic Research (project No. 15-34-20864 and 16-34-00415).

\section{REFERENCES}

1. Perou CM, Sorlie T, Eisen MB, et al. Molecular portraits of human breast tumours. Nature 2000; 406: 747-52.

2. Parker JS, Mullins M, Cbeang MC, et al. Supervised risk predictor of breast cancer based on intrinsic subtypes. J Clin Oncol 2009; 27: 1160-7.

3. Dieci MV, Orvieto E, Dominici M, et al. Rare breast cancer subtypes: histological, molecular, and clinical peculiarities. Oncologist 2014; 19: 805-13.

4. Hugh J, Hanson J, Cheang MCU, et al. Breast cancer subtypes and response to docetaxel in node-positive breast cancer: use of an immunohistochemical definition in the BCIRG 001 trial. J Clin Oncol 2009; 27: 1168-76.

5. Yanagawa M, Ikemot K, Kawauchi S, et al. Luminal A and luminal B (HER2 negative) subtypes of breast cancer consist of a mixture of tumors with different genotype. BMC Research Notes 2012; 5: 376.

6. Cheang MC, Chia SK, Voduc D, et al. Ki67 index, HER2 status, and prognosis of patients with luminal B breast cancer. J Natl Cancer Inst 2009; 101: 736-50.

7. Holm K, Hegardt C, Staaf J, et al. Molecular subtypes of breast cancer are associated with characteristic DNA methylation patterns. Breast Cancer Res 2010; 12: 36.

8. Theillet C. What do we learn from HER2-positive breast cancer genomic profiles? Breast Cancer Res 2010; 12: 107.

9. Carey LA, Dees EC, Sawyer L, et al. The triple negative paradox: primary tumor chemosensitivity of breast cancer subtypes. Clin Cancer Res 2007; 13: 2329-34.

10. Hirshfield KM, Ganesan S. Triple-negative breast cancer: molecular subtypes and targeted therapy. Curr Opin Obstet Gynecol 2014; 26: 34-40.

11. Livasy CA, Karaca G, Nanda R, et al. Phenotypic evaluation of the basal-like subtype of invasive breast carcinoma. Mod Pathol 2006; 19: 264-71.

12. Hoadley KA, Weigman VJ, Fan C, et al. EGFR associated expression profiles vary with breast tumor subtype. BMC Genomics 2007; 8: 258.

13. Laakso M, Tanner M, Nilsson J, et al. Basoluminal carcinoma: a new biologically and prognostically distinct entity between basal and luminal breast cancer. Clin Cancer Res 2006; 12: 4185-91. 
14. Perelmuter VM, Zavyalova MV, Vtorushin SV, et al. Hematogenous metastasis depending on histologic tumor pattern in breast cancer. Siber J Oncol 2006; 19: 29-33.

15. Zavyalova MV, Denisov EV, Tashireva LA, et al. Phenotypic drift as a cause for intratumoral morphological heterogeneity of invasive ductal breast carcinoma not otherwise specified. BioResearch Open Access 2013; 2: 148-54.

16. Zavyalova MV, Perelmuter VM, Vtorushin SV, et al. The presence of alveolar structures in invasive ductal NOS breast carcinoma is associated with lymph node metastasis. Diagn Cytopathol 2013; 41: 279-82.

17. Denisov EV, Gerashchenko TS, Zavyalova MV, et al. Invasive and drug resistant expression profile of different morphological structures of breast tumors. Neoplasma 2015; 62: 405-11.

18. Toft DJ, Cryns VL. Minireview: basal-like breast cancer: from molecular profiles to targeted therapies. Mol Endocrinol 2011; 25: 199-211.

19. Perelmuter VM, Zavyalova MV, Vtorushin SV, et al. Genetic and clinic-pathological characteristics of breast can- cer in premenopausal and postmenopausal women. Uspehi Gerontologii 2008; 21: 643-53 (in Russian).

20. Ahn SH, Kim HJ, Lee JW, et al. Lymph node ratio and $\mathrm{pN}$ staging in patients with node-positive breast cancer: a report from the Korean breast cancer society. Breast Cancer Res Treat 2011; 130: 507-15.

21. Vinh-Hung V, Joseph SA, Coutty N, et al. Age and axillary lymph node ratio in postmenopausal women with T1T2 node positive breast cancer. Oncologist 2010; 15: 1050-62.

22. Chue KM, Yong WS, Thike AA, et al. Predicting the likelihood of additional lymph node metastasis in sentinel lymph node positive breast cancer. Validation of the Memorial Sloan-Kettering Cancer Centre (MSKCC) Nomogram. J Clin Pathol 2014; 67: 112-9.

23. Van la Parra RFD, Peer PGM, Ernst MF, et al. Metaanalysis of predictive factors for non-sentinel lymph node metastases in breast cancer patients with a positive SLN. Eur J Surg Oncol 2011; 37: 290.

24. Paget $S$. The distribution of secondary growths in cancer of the breast. Cancer Metastas Rev 1989; 8: 98-101. 